\title{
Surgical Audit of Sino-Nasal Diseases in a Tertiary Care Centre-A Prospective Study
}

\author{
Ganesh Kumar Balasubramanian, Ramanathan Thirunavukkarasu, \\ Ramesh Babu Kalyanasundaram, Gitanjali Narendran \\ Thanjavur Medical College, Thanjavur, India \\ Email: drganeshkumarb@gmail.com
}

Received 22 July 2015; accepted 26 September 2015; published 29 September 2015

Copyright (C) 2015 by authors and Scientific Research Publishing Inc.

This work is licensed under the Creative Commons Attribution International License (CC BY). http://creativecommons.org/licenses/by/4.0/

(c) () Open Access

\begin{abstract}
Aims of the study: 1) To study the distribution of various Sino-nasal diseases in our region; 2) To emphasize the significance of Functional Endoscopic Sinus Surgery. Material and methods: This surgical study was carried out in the Department of Otorhinolaryngology and Head and Neck Surgery, Thanjavur Medical College, Thanjavur. The study period was from $21^{\text {st }}$ March 2014 to $20^{\text {th }}$ March 2015 (267 cases). After a preliminary examination, all patients were subjected to Diagnostic Nasal Endoscopy followed by radiological examination. Based on the symptoms and the findings, patients were treated by surgery (mainly FESS), pharmacotherapy, and chemo-radiotherapy. Results: In our study, the majority of cases were deviated nasal septum with sinusitis about $62 \%$ (165 cases). The other cases were antrochoanal polyp 8\% (21 cases), ethmoidal polyposis $9 \%$ ( 23 cases) and others $21 \%$ (51 cases). The age group vulnerable for these diseases is 20 - 40 years. Males are affected more commonly than females. Conclusion: Majority of the cases were treated by Functional Endoscopic Sinus Surgery. So we have to improve the surgical skills in this field to achieve a good outcome.
\end{abstract}

\section{Keywords}

Pure Tone Audiogram, Conductive Deafness, Sudden Sensorineural Hearing Loss, Ototoxicity, Presbyacusis, Noise Induced Hearing Loss

\section{Introduction}

This method of auditing the surgeries done in our department is a systematic and proper scrutiny of the quality of service provided to the society to improve our skills, to teach our postgraduates and also to create awareness among the public regarding the morbidity due to sinonasal diseases in our area. The nose is a prominent struc-

How to cite this paper: Balasubramanian, G.K., Thirunavukkarasu, R., Kalyanasundaram, R.B. and Narendran, G. (2015) Surgical Audit of Sino-Nasal Diseases in a Tertiary Care Centre-A Prospective Study. International Journal of Otolaryngology and Head \& Neck Surgery, 4, 369-374. http://dx.doi.org/10.4236/ijohns.2015.45061 
ture in the face with aesthetic and functional significance. It is commonly affected by allergy. The most common symptoms related to these sinonasal disorders are nasal obstruction followed by nasal discharge, disturbance of smell, bleeding from the nose, headache, facial swelling, change of voice, visual disturbances, epiphora and mouth breathing [1].

According to the National centre of Disease Statistics, sinusitis has become the most common chronic illness in the United States, surpassing arthritis. Sinonasal disease may exacerbate asthma and other Chronic Obstructive Pulmonary Diseases [2]. In developing countries like India, allergy is a common problem and most commonly seasonal. So assessment of the type and severity of allergy is mandatory to control the chronic allergic problems related to nose and paranasal sinus disorders.

Polyp is any mass of tissue that bulges or projects downwards from surface and is visible macroscopically. It is defined as prolapsed, pedunculated, polypoidal paranasal sinus mucosa. Hippocrates described the polyp as early as 460 - 370 B.C. He is considered as "Father of Rhinology". Mechanical obstruction produced by nasal septal deviation will produce persistent infection of the paranasal sinuses. So, septal surgery is necessary for these cases. Messerklinger highlighted that Osteo Meatal Complex (OMC) is the basic unit responsible for the development of infection in frontal, maxillary and ethmoid sinuses. Stammberger, Kennedy and Messerklinger also stated that cilia beated towards the natural ostium. Frontal and ethmoid sinuses drain into the middle meatus via anterior ethmoid and hence their physiology and pathology depend on conditions of the anterior ethmoid [3].

Rhinosporidiosis is managed by excision of the mass and diathermy cauterization of the base to reduce recurrence. Nasolabial cysts are removed by sublabial approach. Nasal bone reduction surgery is done by using Asche and Wallsham forceps. Biopsies of maxillary sinus malignancy were taken through inferior meatal antrostomy. These patients underwent maxillectomy. Nasopharyngeal carcinoma cases were subjected to radiotherapy after DNE and biopsy.

\section{Materials and Methods}

This prospective study was carried out in the Department of Otorhinolaryngology and Head and Neck Surgery in Thanjavur Medical College, Thanjavur. The study period was from March $21^{\text {st }} 2014$ to March $20^{\text {th }} 2015$ (one year). Total number of cases taken up for this audit was 267 cases. All these cases were examined by the consultants and subjected to diagnostic nasal endoscopy and computed tomography. We used Hopkins Rod endoscopes and Karl-Storz cold light source system for the Functional Endoscopic Sinus Surgery. We used appropriate angled scopes for the surgery of maxillary and frontal sinuses. All malignancy cases underwent biopsy first. Depending upon the histopathological report, definite treatment was planned. Post operative follow up is the mainstay for better outcome for all the endoscopic sinus surgeries. All the allergy related diseases were managed initially with systemic steroids tapered gradually and maintained with inhalational steroids.

\section{Results}

In our study Deviated Nasal Septum with Sinusitis cases are more in number, about 165 cases (62\%). The sinusitis is mostly chronic and some are due to allergy. In cases with marked septal deviation, the opposite inferior turbinate will be hypertrophied. This is due the presence of submucosal neurovascular tissue in the inferior turbinate alone. The ethmoidal polyp cases are around 23 in number (9\%). These polyps are formed due to allergy. Antrochoanal polyp cases are 21 in number (8\%). Infection is the main etiology for this polyp followed by allergy. The others contribute about $21 \%$ (58 cases) (Chart 1 ).

DNS with Sinusitis is more common in the 20 - 40 years age group. These age groups are more commonly affected by allergy due to greater exposure to polluted environment. We know very well that antrochoanal polyp is more prevalent in children and adolescents. Our study also shows that 5 - 19 years is more commonly affected by antrochoanal polyp. It is rare in older age (over 50 years). But the reverse is true for ethmoidal polypi which are more common in the age group of 40 - 60 years, and very rare in younger age groups (Table 1 and Chart 2).

Males are more commonly affected by DNS with Sinusitis than the females. Antrochoanal polyp more commonly involves females than the males. Ethmoidal polyp equally affects both sexes (Table 2 and Chart 3).

The other cases are as follows:

Nasolabial cyst ( 8 cases) -5 were left sided cysts and the remaining 3 were right sided. There were 7 cases of rhinosporidiosis, 3 cases of juvenile nasopharyngeal angiofibroma, nasopharyngeal carcinoma 4 cases, 5 cases of benign tumors of the nasal cavity, 5 cases of carcinoma of the maxillary sinus, 3 cases of bleeding polypus of 


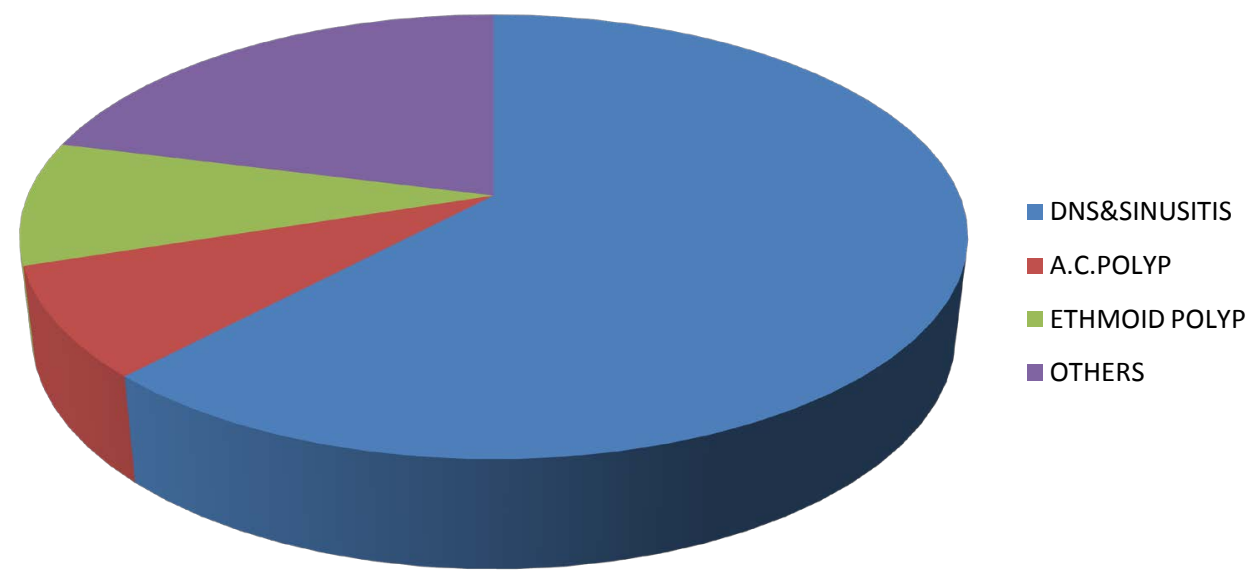

Chart 1. Distribution of common sinonasal diseases.

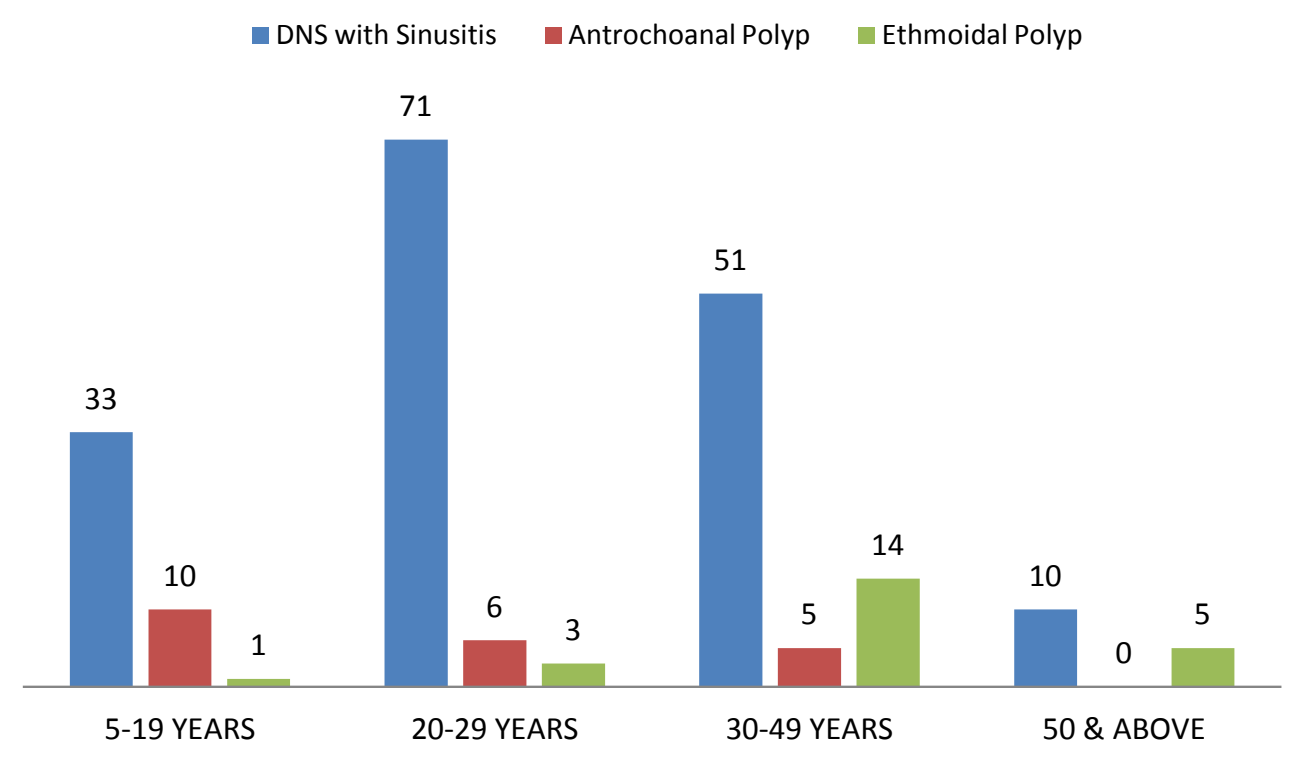

Chart 2. Common nasal pathologies in different age groups.

MALE IFEMALE

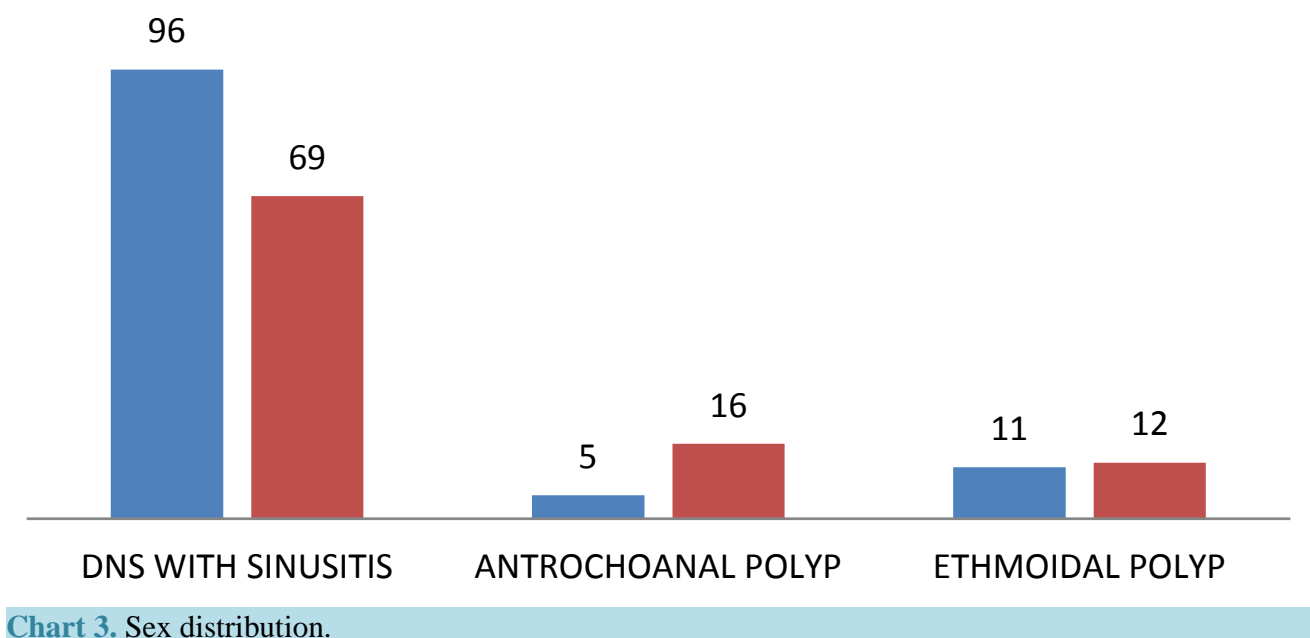

Chart 3. Sex distribution. 
Table 1. Age distribution of DNS and polyp cases.

\begin{tabular}{ccccc}
\hline Age Group & DNS with Sinusitis & Antrochoanal Polyp & Ethmoidal Polyp & Total Cases \\
\hline 5 - 19 years & 33 & 10 & 1 & 44 \\
20 - 29 years & 71 & 6 & 3 & 80 \\
30 - 49 years & 51 & 5 & 14 & 70 \\
50 \& above & 10 & 0 & 5 & 15 \\
\hline
\end{tabular}

Table 2. Sex distribution.

\begin{tabular}{cccc}
\hline Type of Diseases & MALE & FEMALE & TOTAL \\
\hline DNS with Sinusitis & 96 & 69 & 165 \\
Antrochoanal Polyp & 5 & 16 & 21 \\
Ethmoidal Polyp & 11 & 12 & 23 \\
\hline
\end{tabular}

septum, and 5 cases of fungal sinusitis, one case of cerebrospinal fluid Rhinorrhea, 3 cases of nasal myiasis, 4 cases of Rhinolith and 10 cases of fracture of nasal bone.

\section{Discussion}

Deviated nasal septum and sinusitis disturbs the normal function of the sinuses such as ventilation and drainage. It commonly causes nasal obstruction, epistaxis. For complete relief from sinusitis, we have to remove the disease, reduce the tissue odema, facilitate drainage and maintain permeability of ostia. Only then we can achieve normal aeration and ventilation of sinuses [4]. In the nineteenth century Caldwell-Luc surgery was the treatment of choice for Maxillary sinusitis. But nowadays it is replaced by FESS. Medical management of sinusitis has little benefit in patients with chronic refractory sinusitis with an underlying immunodeficiency, for that group we have to do combined management of surgical drainage and medical management [5].

Chronic sinusitis is increasing in number in developing countries like India. Sinusitis presents with headache, nasal discharge and postnasal drip. Treatment is initially medical management and surgery is done if there is no improvement with medicines. In 1901 Hirschmann used modified Nitze cystocsope to examine sinuses. Speilberg was the first person to use an endoscope to examine the maxillary sinus through inferior meatus. Maltz coined the term sinuscopy. The development of compact, straight and various degrees of angled endoscopes and the pioneering work of Messerklinger and Wigand et al revolutionised FESS. In 1985 William Kennedy coined the term FESS. Stammberger, Kennedy and Zinreich (1996) contributed for the widespread use of endoscopic diagnosis and management for sino-nasal diseases [6].

In our study majority of patients belong to 20 - 49 years which coincides with Rahman et al. but is inconsistent with Venkatachalam. The main presenting symptoms include nasal obstruction, nasal discharge, headache, facial pain, post nasal drip and recurrent sore throat. This is similar to Rice and Mathews et al.'s studies [7].

Sino-nasal polyps are more common in females compared to males in our study, in contrast to the Zafer et al. Indian study which shows male preponderance [8]. Antro choanal polyp otherwise called as Killian's polyp is common in adolescent age and in children. But ethmoidal polyp is more common in elderly. Recurrence is common in ethmoidal polyp. Malignant transformation is common in ethmoidal polyp. Nasal polyps result from chronic inflammation of the mucosa of the nose and sinuses. The symptoms are nasal obstruction, nasal discharge and change of voice. There is a strong association with asthma, aspirin sensitivity, nasal polyp (Samter's Triad) [9].

Commonest site of origin of AC polyp is floor and posterolateral wall of maxillary sinus. So it is difficult to remove it completely from its attachment in routine endoscopic sinus surgeries. In case of recurrence of AC polyp, treatment of choice is Caldwell-Luc approach, because we can easily reach the floor and posterolateral wall. All ethmoidal polyp cases were treated by oral steroids preoperatively then taken up for surgery and postoperative topical steroids were given to reduce the recurrence rate [10]. We used powered instruments like microdebrider for ethmoidal polyposis and in cases of hypertrophied inferior turbinate. 
Bleeding polypus of septum is capillary haemangioma. The patient presents with epistaxis. It mostly arises from cartilaginous part of the septum. Cavernous type is rare in sino-nasal tract [11]. Inverted papilloma is a benign tumour but recurs if not treated properly and adequately. Late recurrence even after 30 years is common. Malignant transformation is about $11 \%$ (squamous cell carcinoma) [12].

Malignancy of sinonasal tract is rare. Maxillary sinus carcinoma is common. The patient complains of swelling or numbness in cheek, nasal obstruction, epistaxis, visual disturbances, facial pain, loose teeth or swelling in palate. The most common histological type is squamous cell carcinoma, commonly seen after the $4^{\text {th }}$ decade. All tumours of maxillary sinus erode the lateral nasal wall [13]. Complete surgical resection of tumor followed by adjuvant radiotherapy improves survival rate [14].

In our study there were 8 cases of nasolabial cyst. Among this 5 cases were left sided cysts and the remaining 3 were right sided. They were removed in to by sublabial approach and followed up. One case recurred. There were 7 cases of Rhinosporidiosis. It was treated by excision and cauterization of the base using bipolar diathermy. We saw recurrence in 2 cases, in which the rhinosporidial mass presented in the inferior meatus, which is very difficult to remove completely due to minimal available space.

We operated 3 cases of Juvenile Nasopharyngeal Angiofibroma which was identified in the early stage and we completely removed the mass. We faced minimal bleeding only because of predominance of fibrous element. Nasopharyngeal carcinoma (4 cases) underwent DNE and biopsy taken from the fossa of rosenmuller which was obliterated in all the cases. All the cases were diagnosed as squamous cell carcinoma and sent for Radiotherapy and are under regular follow up.

There are 5 cases of benign tumors of the nasal cavity in our study. After biopsy all the cases underwent surgery for complete removal of the masses. For 2 cases we removed the mass by Lateral Rhinotomy approach and the others by trans nasal route. Around 5 cases of Carcinoma of the Maxillary sinus are presented in our study. They were subjected to biopsy after Inferior Meatal Antrostomy and after that 3 cases underwent total maxillectomy and 2 cases were sent for Brachytherapy. There are 3 cases of bleeding polypus of septum in our study which were excised. About 5 cases of fungal sinusitis are seen in our study. They were confirmed by biopsy and specific antifungals were given according to culture and sensitivity. Surgical removal of the fungal mass was done endoscopically.

Cerebrospinal fluid rhinorrhea was treated endoscopically by sandwich method using cartilage, fat, temporalis fascia, muscle, surgicel and mucosal flaps. The site of leakage was medial lamellae of cribriform plate. 3 cases of nasal myiasis were treated medically by using tincture benzoin and ether. All 3 patients were in immunocompromised state. Rhinolith were removed endoscopically. Fracture of nasal bone was treated by nasal bone reduction surgery using various forceps. Two patients underwent septorhinoplasty.

\section{Conclusions}

With appropriate history, nasal endoscopy and proper radiological imaging correct diagnosis can be made and proper treatment can be given. Nasal endoscopy is the best diagnostic test. FESS is a safer, efficient procedure for sinonasal diseases. Because of better illumination of the surgical field, we can achieve the best results with minimal or nil complication.

This study helps us to identify the distribution of various types of sino-nasal diseases and we can train doctors in surgical techniques and educate the patients regarding the mode of spread of certain diseases like Rhinosporidiosis. We can advise the nasal polyp cases for regular postoperative follow-up and steroid management.

\section{References}

[1] Humayun, A.H.M., Zahurul Huq, A.H.M., Ahmed, S.M.T., et al. (2010) Clinicopathological Study of Sinonasal Masses. Bangladesh Journal of Otorhinolaryngology, 16, 15-22. http://dx.doi.org/10.3329/bjo.v16i1.5776

[2] Levine, H.C. (1990) Functional Endoscopic Sinus Surgery: Evolution, Surgery and Follow up of 250 Patients. Laryngoscope, 100, 79-84.

[3] Stammberger, H. (1986) Endoscopic Endonasal Surgery Concepts in the Treatment of Recurring Rhinosinusitis. Part I. Anatomic and Patho-Physiologic Considerations. Otolaryngology_Head \& Neck Surgery, 94, 143-147.

[4] Park, A.H., Lau, J., Stankiewicz, J. and Chow, J. (1998) The Role of Functional Endoscopic Sinus Surgery in Asthmatic Patients. Journal of Otolaryngology, 27, 275-280.

[5] Buehring, I., Friedrich, B., Schaaf, J., Schmidt, H., Ahrens, P. and Zielen, S. (1997) Chronic Sinusitis Refractory to 
Standard Managements in Patients with Humoral Immunodeficiencies. Clinical \& Experimental Immunology, 109, 468-472. http://dx.doi.org/10.1046/j.1365-2249.1997.4831379.x

[6] Stankiewicz, J. (1991) Endoscopic Nasal and Sinus Surgery. Surgery of the Para Nasal Sinuses. $2^{\text {nd }}$ Edition, W.B. Saunders Company, Philadelphia, 223-224.

[7] Mathews, B.L., Smith, L.E., Jhones, R., Miller, C. and Bookschmidt, J.K. (1991) Endoscopic Sinus Surgery: Outcome in 155 Cases. Otolaryngology - Head \& Neck Surgery, 104, 244-246.

[8] Zafar, U., Khan, N., Afroz, N., et al. (2008) Clinico Pathological Study of Non Neoplastic Lesions of Nose and Paranasal Sinuses. Indian Journal of Pathology \& Microbiology, 51, 26-29.

[9] Hedman, J., Kaprio, J., Poussa, T., et al. (1999) Prevalence of Asthma, Aspirin Intolerance, Nasal Polyposis and COPD in a Population Based Study. International Journal of Epidemiology, 28, 717-722. http://dx.doi.org/10.1093/ije/28.4.717

[10] Dassonville, C., Bonfils, P., Momas, I., et al. (2007) Nasal Inflammation Induced by a Common Cold: Comparison between Controls and Patients with Nasal Polyposis under Topical Steroid Spray. Acta Otorhinolaryngologica Italica, 27, 78-82.

[11] Pradhanaga, R.B., Adhikari, P., Thapa, N.M., et al. (2008) Overview of Nasal Masses. J Inst Med, 30, 13-16.

[12] Barnes, L., Tse, L.L.Y. and Hunt, J.L. (2005) Schneiderian Papillomas. In: WHO Classification of Tumours, Lyon: Pathology of Head and Neck Tumours, IARC Press, Lyon, 28-32.

[13] Fasunla, A.J. and Lasis, A.O. (2007) Sinonasal Malignancies: A 10-Year Review in a Tertiary Health Institution. Journal of the National Medical Association, 99, 1407-1410.

[14] Hoppe, B.S., Stegman, L.D., Zelefsky, M.J., et al. (2007) Treatment of Nasalcavity and Paranasal Sinus Cancer with Modern Radiotherapy Techniques in the Postoperative Setting-MSKCC Experience. International Journal of Radiation Oncology • Biology • Physics, 67, 691-702. http://dx.doi.org/10.1016/j.ijrobp.2006.09.023 